\title{
TELECONEXIONES CLIMÁTICAS CON INFLUENCIAS SOBRE ALGUNOS RÍOS DEL SUDESTE DE EUROPA
}

\author{
Dumitru Mihăilă, \\ Andrei-Emil Briciu \\ Departamento de Geografía. Universidad 'Ştefan cel Mare'. Rumania \\ andreibriciu@atlas.usv.ro
}

Diversos fenómenos climáticos de ciertas zonas del globo han resultado ser la causa de algunas manifestaciones climáticas e hidrológicas de otras zonas, situadas a una considerable distancia de la zona de origen de los fenómenos. Hemos examinado la zona compartida entre Rumania, Ucrania y la República de Moldavia, con una gran densidad de fuentes de datos con el fin de identificar la existencia de ciertas influencias externas de la zona del estudio sobre el clima y la hidrología regional y asimismo las variaciones espacio-temporales de estas influencias.

El territorio geográfico investigado ocupa una superficie de $45000 \mathrm{~km}^{2}$, en el Noreste de Rumania, la extremidad del Oeste de la República de Moldavia y el Sudeste de Ucrania, siendo enmarcado entre las altitudes de $2100 \mathrm{~m}$ y $3 \mathrm{~m}$.

Han sido utilizados datos relativos al fluir de los ríos (F) de 45 estaciones hidrométricas. Las cuotas medias anuales del fluir de los ríos han variado de $6388 \mathrm{~m}^{3} \mathrm{~s}^{-1}$ a DC, $213.6 \mathrm{~m}^{3}$ $\mathrm{s}^{-1}$ a SL, $111.9 \mathrm{~m}^{3} \mathrm{~s}^{-1}$ a PD (el más grade fluir analizado) a $1 \mathrm{~m}^{3} \mathrm{~s}^{-1}$ la $\mathrm{BM}, 0,18 \mathrm{~m}^{3} \mathrm{~s}^{-1}$ a BT, $0,81 \mathrm{~m}^{3} \mathrm{~s}^{-1}$ a BE. La media del fluir de los ríos en los sectores monitorizados ha sido de 176.4 $\mathrm{m}^{3} \mathrm{~s}^{-1}$ incluyendo el fluir del Danubio y $35.2 \mathrm{~m}^{3} \mathrm{~s}^{-1}$ sin tomar en calculo el Danubio. Las mayorías de las estaciones hidrológicas han tenido datos del periodo entre 1950 y 2011. Las estaciones BT, BE y BM han tenido datos de 1950 hasta incluso 2007. BC, MF, MI, MD, OL, OD, SH y SN han representado datos entre 1950 y 2009 incluso. Hacen excepción DC (1950-2008), SC (1950-1995), ST (1991-2009), SO (1953-1995), OP (1979-2011), PD y PU (1975-2005) y PP (1981 -2005).

Para los análisis de las particularidades climáticas regionales hemos utilizado datos mensuales referentes a la temperatura del aire $(\mathrm{T})$ y a las precipitaciones atmosféricas $(\mathrm{P})$ disponibles en la base de datos CRU, representando medios para regiones de $0.5 \times 0.5$ grados latitud y longitud. 39 regiones CRU como estas han cubierto casi en totalidad la superficie investigada. Los datos CRU han sido utilizados para el intervalo disponible entre 1950-2009 y han sido preferidas porque cubren uniformemente la región con valores reales interpolados (de las estaciones meteorológicas) en función de altimetría. 
Los datos para los índices climáticos estandarizados (NAOI, AOI, SOI) han sido obtenidos de NOAA y han sido utilizados como principales indicadores teleconectivos.

La dirección principal del presente estudio quiere evidenciar los factores climáticos mayores con implicaciones hidrogeográficas y argumentar las influencias hidrológicas del 2cSP (los principales dos cuencas hidrográficas de los ríos Siret y Prut) incluidos inducido por NAO, AO y SO. Otra dirección del presente estudio queda abierta a unas futuras investigaciones e identifica las diferencias de régimen hidrológico de la zona investigada con el fin de examinar los flujos y las tendencias evolutivas bajo-regionales y cuantificar las influencias teleconectivas sobre los flujos.

Aunque para la primera dirección el algoritmo de trabajo ha sido simple para la segunda tenemos que explicar en detalle los pasos. Con el fin de identificar las arias hidrológicas representativas de la media F y los valores estandarizados los coeficientes de correlación Pearson (r) han sido calculados en primer lugar para todas las estaciones hidrométricas. Todas las correlaciones han sido positivas. Las estaciones con una correlación $\geq 0,8$ (fuerte) con otras estaciones han sido seleccionadas de la matriz de correlación de todos los ríos (DC, la única estación del Danubio ha sido eliminada de los ulteriores cálculos porque para este sector el Danubio no ha tenido correlaciones fuertes con los ríos del 2cSP). Estas estaciones han sido marcadas en los mapas intermediarios de trabajo utilizadas para obtener las últimas seis arias hidrológicas usadas para obtener las últimas seis arias hidrológicas usadas para correlacionar el parámetro F con otros parámetros. Cada mapa intermediario incluye las estaciones de una cierta cuenca hidrográfica que son fuertemente correlacionadas entre sí, así como también otras estaciones situadas en diferentes cuencas hidrográficas, con las que estas tienen fuertes correlaciones.

Con el fin de elegir las cuencas hidrológicas para los mapas intermediarios han sido utilizados los principales ríos de la zona investigada (los ríos: Siret, Suceava, Moldova, Ozana, Bistriţa, Trotuş, Putna, Milcov, Râmnic, Bârlad, Prut, Jijia, Bahlui y Bahlueţ) en las que han sido marcadas todas las estaciones hidrométricas estudiadas. Como todas las estaciones relevantes en los mapas intermediarios han tenido conjuntos de datos, la intersección de conjuntos ha sido utilizada para obtener las arias hidrológicas finales con un comportamiento hidrológico similar para las estaciones constituyentes. De esta manera, por ejemplo, si el punto A presenta una fuerte correlación $(\geq 0.8)$ con el punto B y si el punto B presenta una fuerte correlación con el punto $\mathrm{C}$, pero el $\mathrm{C}$ no presenta una fuerte correlación con A sino con el punto D que tiene correlación con A y con B, entonces los puntos A, B y D forman una aria hidrológica mientras que el punto $\mathrm{C}$ queda aislado o formará parte de otra aria. De esta manera, las estaciones hidrológicas han formado arias hidrológicas sólo si cada estacion hidrológica pertenente a una aria hidrológica ha presentado fuertes correlaciones con todas las estaciones de la misma aria hidrológica. 29 estaciones (64.4\%) de las 45 existentes han presendado correlaciones semejantes. Los puntos aislados y los puntos que tienen correlaciones. Los puntos aislados y los que tienen correlaciones bajo 0.8 han sido utilizados para marcar lo más preciso posible las fronteras entre las provincias hidrológicas. Teniendo en cuenta que las estaciones de la misma cuenca hidrológica tienen a menudo correlaciones más de 0.7 , la distribución de las estaciones restantes hacia una aria hidrológica u otra ha sido muy fácil de resolver.

Entre el comportamiento climático e hidrológico de los territorios de las áreas hidrológicas prevalecen las concordancias, ciertos elementos de discordancia subrayando finalmente la identidad hidrológica de las subunidades identificadas. 
Hemos analizado la correlación de las temperaturas, de las precipitaciones atmosféricas y del fluir medio de la región entera con NAOI, AOI y SOI.

Se establecen correlaciones relevantes entre P y NAOI, respectivo entre P y AOI. Las matrices de correlación muestran una fuerte correlación negativa entre los índices y las cantidades de precipitaciones en la temporada de frío. Más que esto hay un cambio gradual y relativamente la constante de signo entre la temporada de frío y el verano.

El verano muestra correlaciones extremamente bajas, positivas, que indican que las dos oscilaciones del hemisferio nórdico afectan en clima regional en la temporada de frio. Las correlaciones medias anuales para la zona entera equivalen con -0.24 para AOI- P y -0.14 para NAOI-P. Se puede observar que la determinación de las correlaciones medias anuales de la zona entera a base de la mediación de las correlaciones medias mensuales por arias hidrológicas genera un coeficiente de correlación entre los parámetros de toda la región más grande que la simple correlación de dos meses medios consecutivos para la región entera.

Las correlaciones anuales desvanecen una oscilación estacional de la intensidad de la correlación. Se nota la existencia de dos cumbres de influencia ZOI y NAOI en la temporada fría (Tabla 4). El análisis de la correlación entre AOI- F y NAOI-F muestra que estas tienen casi la misma evolución durante el año y por regiones, pero las influencias AOI y NAOI son más débiles sobre el F que sobre el P: con $20 \%$ más débiles para F-AOI y unos $14 \%$ más débiles para F-NAOI. Las más fuertes modificaciones se producen en enero y febrero (-46\% y $45 \%$ para la influencia AO y $-31 \%$ si $-34 \%$ para la influencia NAO). Además la amplitud entre el máximo y el mínimo de las modificaciones de las influencias de las oscilaciones sobre $\mathrm{P}$ y F es de 1.8 veces más grande en el caso de AOI. Podemos destacar el siguiente aspecto: cuanto más fuerte es la influencia de una oscilación (AO) cuanto más variable es la evolución de valor del índice aferente (en nuestro caso AOI).

Entre la estadística de Topor y Stoica de 1965, basada en el análisis del relieve del campo bárico a nivel europeo en el periodo 1938-1962 y las obtenidas por nosotros, basadas en los índices estandarizados de la AO y F se pueden hallar similitudes y diferencias. Las semejanzas residen en la gran importancia que ambos estudios conceden a la circulación polar y los AO. Las diferencias derivan de los periodos diferentes a los que se refieren los dos estudios (1895-1955 y 1950-2009, respectivamente) y la metodología diferente con que se ha abordado la circulación polar/oscilación ártica. Topor y Stoica (1965) han establecido una ponderación multianual, mientras que nuestro estudio no es sinóptico, sino tiene como eje los valores mensuales, -y a partir de aquí, multianuales, periódicos, referidos a las cuatro estaciones del año-, estandarizados de las AO. Esto lleva a una ponderación más baja en el estudio de referencia de los casos de covariación (AOI-)-(F-) y (AOI)-(F+) en comparación con el estudio al que nos referimos, que a nuestro parecer son mayoritariamente el atributo de la circulación polar directa y ultrapolar (32\% a nivel anual, $43 \%$ en el caso del semestre frío y $53 \%$ en los meses de invierno), es comparable con, aunque más bajo, que el del 58,3\% calculado por Topor y Stoica (1965) para los casos de circulación polar. (AOI-)-(F+) que en el estudio de Torpor y Stoica representan la circulación polar devuelta, la cual en el estudio de Topor y Stoica (1965) alcanza al $41 \%$ de los casos, en nuestro análisis se sitúan respectivamente entre el 16 y $17 \%$ a nivel anual -estación fría -invierno. La covariante AOI-/F- es la más significativa ligadura como nivel de relevancia establecida entre $\mathrm{AO}$ y $\mathrm{F}$ en $2 \mathrm{cSP}$. 
Midiendo los valores SOI del tiempo de los episodios El Niño (en número de 13, con la atención puesta en el intervalo enero-marzo, en el cual la oscilación SO alcanza su apogeo) y los índices estandarizados de precipitaciones (P) y caudales (F) de 2cSP hemos observado que, en semejante intervalo, tanto precipitaciones como caudales se sitúan debajo de lo normal. Es posible que este tipo de teleconexiones (SOI-)-(P-), (SOI-)-(F-) no exista en una forma tan pronunciada como la que muestra la situación estadística. Es posible que en esta situación estemos hablando solo de una coincidencia estadística que esconde otros mecanismos teleconectivos o la influencia de los factores climatogénicos locales/regionales. Esta constatación es reforzada también por la estadística de las correlaciones (SOI+)-(P), (SOI+)(F) casos de covariación en 2cSP. La covariación mensual entre SOI y F (720 casos han sido tenidos en consideración) es muy débil si se expresa en cualquier escala temporal. No hemos podio distinguir un mecanismo teleconectivo claro entre SO y F en 2cSP. Mucho más que eso, estos casos no se pliegan a ninguna de las realidades teleconectivas cuyas consecuencias son conocidas y explicadas para otras zonas o regiones geográficas.

Hemos examinado la correlación entre las correlaciones de diferentes parámetros, con objeto de observar si estas tienen una evolución al margen de la caída/elevación de los valores. Tal como se ve en el caso de la influencia NAO sobre los caudales de los ríos, algunos índices o parámetros pueden ser relevantes, pero con un valor extremadamente débil, que algunas veces, no puede tener un sentido sino es mostrada una similitud con otro índice / parámetro similar en su manifestación, pero más intenso. Por ejemplo, la correlación d - 0.86 entre TF y P-F indica el hecho de que existe un evolución aproximadamente asincrónica entre el decurso de un año medio (12 valores mensuales) de la correlación entre T y F y la evolución entre P y F: cuando la temperatura crece, cae la correlación con los caudales porque aparece la evapo(transpira)ción, por el contrario, cuando crecen las precipitaciones, crecen los caudales.

Las correlaciones más fuertes se establecen entre $[\mathrm{F}-\mathrm{AOI}]$ y $[\mathrm{NFAOI}] \div 0,85 \%$. Del mismo modo, podemos deducir que la oscilación próxima (norte - $\mathrm{AO}$, oeste - NAO) acciona sobre el régimen de la corriente preponderante por medio de dos elementos meteorológicos intermediarios: las precipitaciones atmosféricas y la temperatura del aire.

El intento de regionalizar la corriente de 2cSP en base a unos argumentos exclusivamente estadísticos no daría los resultados previstos. En primer lugar porque los factores teleconectivos dejan su huella sobre unos territorios extensos, y su influencia no puede ser detenida en el valle de un río o por el contacto entre una subunidad de llanura y una meseta, entre una meseta y una zona de colinas etc. De entre los factores físico-geográficos, las cadenas montañosas (en nuestro caso los Cárpatos) son capaces de atenuar o suprimir estas influencias, pero la zona completa de estudio está situada sobre la misma parte de los Cárpatos. En este caso las diferencias correlativas entre los índices de las oscilaciones/precipitaciones/caudales de los ríos de cualquiera de las dos superficies del cuadro 2cSP son impuestas por la naturaleza de la superficie activa/topográfica.

Los valores de las correlaciones entre las correlaciones reflejan más bien la medida en que las áreas hidrológicas identificadas responden a la matriz de los factores genéticos responsables de la corriente. El descuido de este hecho exigiría la explicación de algunas sorprendentes diferencias halladas entre los mismo parámetros sobre áreas situadas a muy poca distancia unas de otras (por ejemplo, las correlaciones [F-NAOI]-[P-AOI] tienen un valor 
de 0,17 en el área hidrológica 6 y de 0, 75 en la provincia hidrológica 4 o [F--A]-[P-NAO] tienen un valor de 0,29 en el área hidrológica 6 y de 0,72 en la provincia hidrológica 4 (entre otros muchos casos). Esta tarea es difícil de realizar en el estado actual del conocimiento científico sobre la zona de estudio.

De este modo, El área hidrológica 1 está sometida a cierta influencia escandinavo-báltica (aproximadamente $2 / 3$ de esta provincia: parte central nórdica, norte y noreste). La influencia escandinavo-báltica puede ser atribuida a la circulación polar directa (menos a la circulación ultrapolar) manifestada sobre todo en la estación fría (sobre todo en invierno) en la época de las fases AO negativas, debemos tener en cuanta sin embargo que la parte central occidental, occidental y del sur las influencias oceánicas (por medio de NAO si AO) son importantes.

El área hidrológica 2 está situada en la frontera entre las influencias escandinavo-bálticas (predominantes en al estación fría) y las influencias climáticas continentales (que amplían la influencia escandinavo-báltica durante el invierno), sobre una superficie geográfica exclusivamente de meseta (aprox. $350 \mathrm{~m}$ ) que permite las potentes influencias del anticiclón euroasiático en la estación fría, influencias que afectan, a través de un régimen helada prolongada, el caudal de los ríos, mientras que en la estación cálida, la frecuencia relativamente elevada de los intervalos de tiempo anticiclónico, provoca la caída de los caudales por la falta de precipitaciones.

El área hidrológica 3 tiene un régimen hidrológico influenciado en la misma medida por el clima montañoso, por las advecciones oceánicas, debido a la circulación desde el oeste que alterna con la continental del este (las fases NAO). El predominio de la influencia de un tipo dado impone el carácter pluviométrico e hidrológico de algunos intervalos de tiempo. Las influencias del oeste dan sin embargo la nota dominante de la pluviosidad, gracias a un ligero excedente pluvio-hidrométrico, apoyado también por las características del estrato climático de montaña y de las elevadas colinas (1200-800m), definido en primer lugar por un balance pluvio-hidríco anualmente excedentario.

El área hidrológica 4 tiene un régimen hidrológico intensamente afectado por el poderoso efecto foehn de las masas de aire procedentes del oeste (proceso que alcanza su apogeo en el intervalo de abril-mayo-junio) y algunas veces por la circulación ciclónica retrograda. En invierno son muy importantes las advecciones de masas de aire continental del este (frio y seco). Las masas de aire continental generan unos estados de tiempo, que entran algunas veces en contacto con el aire submediterráneo (caliente y húmedo) generando superficies frontales y sistemas nubosos de las cuales caen nevadas que después se convierten en venticas. Todo esto se desarrolla sobre el fondo de un comportamiento climático predominantemente montañoso en el centro y el oeste, y de llanura baja en el este. Las características de la corriente, impuestas por el espacio montañoso, se prolongan también al espacio inferior de los ríos de la llanura.

El área hidrológica 5 es un área típica de colinas (150-300 m) y de meseta (250-550m) determinada por la influencia de las masas de aire continental., La masas de aire continental son determinante de unos efectos climáticos llenos de contrastes desde el punto de vista térmico, pluviométrico y dinámico. Sobre el fondo general de una pluviometría con deficitaria con el predominio de años, temporadas, estaciones, meses e intervalos de sequía o de precipitaciones escasas, aparecen episodios de precipitación donde la cantidad de lluvia es comparable con la cantidad de lluvia caída en un mes o en un año. Este tipo de intervalos 
con excedente pluviométrico produce picos hidrográficos que generan desbordamientos e inundaciones de efectos catastróficos. La evapora-traspiración alcanza valores muy grandes y los caudales de los ríos se reducen muchísimo, o los ríos son afectados por intensos fenómenos de helada que atenúan o anulan mucho o totalmente el curso del agua. En esta área la influencia ultrapolar es la más evidente.

El área hidrológica 6 la representa solo el régimen del rio Prut en las proximidades del lago Stânca-Costeşti, que impone una poderosa disminución de las influencias montañosas y escandinavo-balticas que encontramos en la corriente del rio Prut a su paso por la provincia 1, por encima del lago. La recepción de unos afluentes del área hidrológica 5 y del territorio de la república de Moldavia (que se encuadra muy probablemente en el mismo régimen del área 5) y el recorrido a través de la república de Moldavia impone al Prut un comportamiento hidrológico híbrido, aparte. Las influencias climáticas continentales y el régimen de montaña representan igualmente factores naturales que intervienen en este caso.

Debido a las distancias tan grandes entre las zonas estudiadas y el reservorio energético de algunas oscilaciones temáticas la influencia de estas por consiguiente sobre 2cSP disminuye aproximadamente en forma directamente proporcional al aumento de las distancias mencionadas, De este modo la influencia SO es más pequeña que la influencia AO. Porque el cuadro natural del 2cSP se comporta como una fuente de ruido en la señal de influencia de los factores externos sobre los valores de las precipitaciones y los caudales, el estudio sobre áreas más reducidas, unitarias desde la perspectiva de los elementos climáticos e hidrológicos es recomendable y útil para la obtención de unas correlaciones más potentes entre lo local y lo global. 